\title{
Vesicles of Toroidal Topology
}

\author{
Udo Seifert \\ Department of Physics, Simon Fraser University, Burnaby, British Columbia, Canada V5A IS6
}

(Received 23 January 1991)

\begin{abstract}
We consider fluid vesicles of toroidal topology. Minimization of the curvature energy at fixed volume and area leads to three different branches of axisymmetric shapes. By using conformal transformations, we identify a large region of nonaxisymmetric shapes in the phase diagram. For vanishing spontaneous curvature, the ground state is twofold degenerate in this region and corresponds to zero pressure difference across the membrane. The relation of these results to the recent observation of toroidal shapes for partially polymerized vesicles is discussed.
\end{abstract}

PACS numbers: $82.70 .-\mathrm{y}, 02.40 .+\mathrm{m}, 46.30 .-\mathrm{i}, 68.15 .+\mathrm{e}$

Two features of fluid membranes allow their characterization in terms of geometrical and topological notions, which contributes essentially to their attractiveness to physicists. ${ }^{1}$ First, their effective two dimensionality arises from a natural separation of length scales. Since a typical membrane has a thickness in the $\mathrm{nm}$ range and typical lateral extensions in the $\mu \mathrm{m}$ range, the membrane can be regarded as a two-dimensional surface embedded in three-dimensional space. Second, fluid membranes have no internal connectivity and, therefore, no elastic energy associated with displacements within the surface. The relevant energy arises solely from the bending of the membrane, which can be translated into curvature of the embedded shape. Closed membranes, i.e., vesicles, can be classified most generally by their topology.

So far, vesicles of spherical topology have been investigated intensively, both experimentally ${ }^{2-7}$ and theoretically. ${ }^{7-16}$ Video microscopy studies reveal an amazing variety of shapes, ${ }^{2,3,7}$ between which transformations can be induced by changing the temperature or the osmotic conditions. Thermally excited shape fluctuations around these low-energy mean shapes have also been analyzed. ${ }^{4-6}$ Both types of experiments support the basic theoretical idea ${ }^{8,9,17}$ that the essential energy is captured by a curvature model. Large regions of the phase diagram have recently been mapped out systematically. ${ }^{15,16}$

In this Letter, we investigate theoretically vesicles of toroidal topology, which have so far only been observed for partially polymerized membranes, ${ }^{18}$ on which we comment at the end. The present study shows, however, that fluid vesicles can also be expected to form such shapes, which should even exhibit qualitatively new features not present for shapes of spherical topology.

Within a minimal model for symmetric bilayers, the energy of a vesicle shape is given by

$$
F \equiv F_{\kappa}+F_{\kappa_{G}} \equiv(\kappa / 2) \oint(2 H)^{2} d A+\kappa_{G} \oint_{K d A},
$$

where $H$ is the mean (extrinsic) and $K$ is the Gaussian (intrinsic) curvature and the integration is over the closed surface. ${ }^{8,9}$ (Here, we have set, for the main part of the paper, a spontaneous curvature $C_{0}=0$, but the influence of a nonzero $C_{0}$ is discussed briefly below.) The two bending rigidities $\kappa$ and $\kappa_{G}$ have the dimension of energy. The (shape) phase diagram for prescribed topology is given by the minimum of $F_{\kappa}$ for fixed area $A$ and enclosed volume $V$. The Gaussian curvature contributes a topological constant given by $F_{\kappa_{G}}=4 \pi \kappa_{G}(1$ $-g$ ), where $g$ is the number of "handles" on the vesicle. The "ground state" (i.e., the shape of lowest energy) is among the solutions of the shape equation,

$$
\delta\left(F_{\kappa}+\Sigma A+P V\right)=0,
$$

which also include saddle points. Here, $\Sigma$ and $P$ are Lagrange multipliers. If a suitable parametrization for the contour of an axisymmetric shape is chosen, this shape equation corresponds to the same four nonlinear first-order ordinary differential equations already introduced for studying vesicles of spherical topology. ${ }^{11,15}$ Only the boundary conditions change in an obvious way.

As solutions to this equation, one finds three branches, which can be characterized according to typical shapes as (i) the sickle-shaped toroids [Fig. 1(a)], (ii) the discoid toroids [Fig. 1(b)], and (iii) the circular toroids [Fig. 1(c)]. Within a branch, the shape varies smoothly

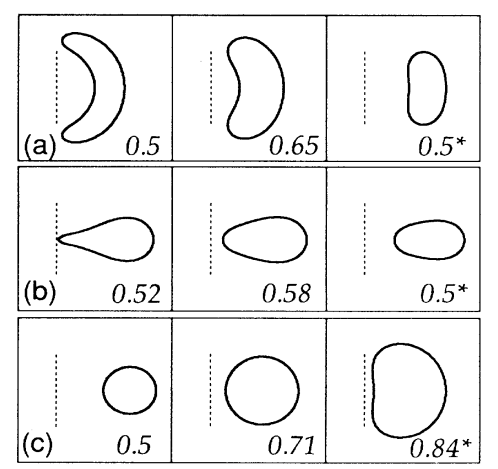

FIG. 1. Cross sections of toroidal shapes for several values of the reduced volume $v$. (a) Sickle-shaped, (b) discoid, and (c) circular toroids. The dashed line denotes the axis of symmetry. Shapes marked with an asterisk are unstable. 
as a function of the reduced volume,

$$
v \equiv V / \frac{4}{3} \pi[A /(4 \pi)]^{3 / 2},
$$

which is the only relevant variable because of the scale invariance of $F_{\kappa}$. In Fig. 2, the bending energy $F_{\kappa}$ of these branches is displayed as a function of $v$.

The branch of sickle-shaped toroids [Fig. 1(a)] exists for $0<v<v_{M}^{\text {sic }}=0.65$. For $v \rightarrow 0$, the contours of the sickles become half circles and the energy $F_{\kappa}$ approaches the value $16 \pi \kappa$ of two perfect spheres. For $v>0$, the energy of these sickle-shaped toroids decreases monotonically and reaches its minimum at the maximal volume $v_{M}^{\text {sic }}$, where the branch develops a cusp. The shapes corresponding to the upper part of the cusp are saddle points, i.e., they are locally unstable with respect to an axisymmetric deformation, as can be derived quite generally by a Landau-type expansion of $F_{\kappa}$ in the vicinity of the cusp. ${ }^{15}$

The discoid branch [Fig. 1(b)] exists for $v_{C}^{\text {dis }}<v$ $<v_{M}^{\text {dis }}$, with $v_{C}^{\text {dis }}=0.50$ and $v_{M}^{\text {dis }} \simeq 0.58$, where this branch also develops a cusp. For $v \rightarrow v_{C}^{\text {dis }}$, the inner diameter of the toroid goes to zero and the shape approaches that of a discocyte vesicle of spherical topology with the north and the south poles touching. This bifurcation of shapes with different topology suggests a mechanism for creating these shapes in the laboratory. Suppose a discocyte vesicle is driven into the range $v \gtrsim v_{C}^{\text {dis }}$, e.g., either by changing the temperature or by osmotic deflation. To create the discoid toroid would, then, only require the formation of a small hole. Although such a process will not occur spontaneously because of the large activation energy required to rupture the membrane, it might be produced by an external probe. ${ }^{19}$

The circular toroids exist for any $0<v<1$. For $v \rightarrow 0$, their shape becomes a torus with exact circular cross section. In this limit, the diameter of the hole vanishes as $1 / v$ and the diameter of the cross section van-

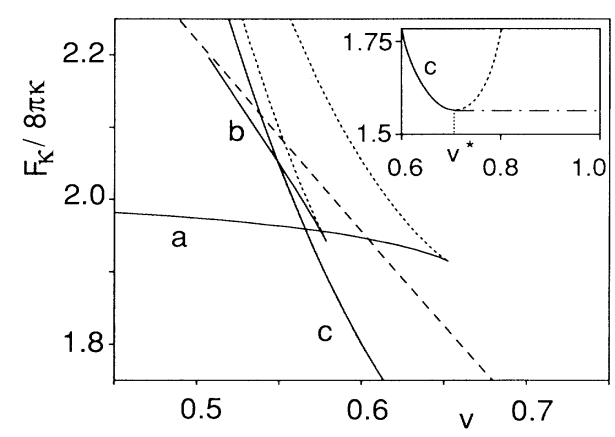

FIG. 2. Curvature energy $F_{\kappa}$ as a function of the reduced volume $v$. Curves $a, b$, and $c$ correspond to Fig. 1 . The dotted parts are unstable. The dashed line denotes the energy of discocyte shapes of spherical topology. Inset: The energy of the circular toroids over a larger range. The dash-dotted line corresponds to conformal transformations of the Clifford torus. ishes as $v$. Consequently, $F_{\kappa}$ diverges as $1 / v^{2}$ in this limit. For $v=v^{*} \equiv 3 / 2^{5 / 4} \pi^{1 / 2} \simeq 0.71$, the bending energy acquires its minimum along this branch with $F_{\kappa}=4 \pi^{2} \kappa$ and the cross section is again perfectly circular. This shape, the so-called Clifford torus, has been known in the mathematical literature before as a solution to (2) for $\Sigma=P=0 .{ }^{20}$ Its shape in Cartesian coordinates can be parametrized by

$$
\mathbf{R}(\theta, \phi)=C((\sqrt{2}+\sin \theta) \cos \phi,(\sqrt{2}+\sin \theta) \sin \phi, \cos \theta),
$$

where $C$ is an arbitrary scale factor and $0 \leq \theta, \phi \leq 2 \pi$ are the internal coordinates. A famous conjecture due to Willmore states that the energy $F_{\kappa}=4 \pi^{2} \kappa$ of the Clifford torus is a lower bound to that of any toroidal shape, irrespective of $v^{20}$ For $v>v^{*}$, the bending energy $F_{\kappa}$ of the axisymmetric circular toroids increases again. These shapes, however, do not correspond to the ground state, as we shall see.

It has recently been recalled ${ }^{21}$ that the bending energy (1) is invariant under conformal transformations (CT's) of the three-dimensional embedding space. ${ }^{20}$ The nontrivial CT's of this space are the inversions in a sphere, i.e., $\mathbf{R} \rightarrow \mathbf{R}_{0}+\left(\mathbf{R}-\mathbf{R}_{0}\right) /\left(\mathbf{R}-\mathbf{R}_{0}\right)^{2}$, where $\mathbf{R}_{0}$ denotes the inversion center. In general, such an inversion fails to conserve the axisymmetry, the area, and the enclosed volume. A simple spatial rescaling (also, a CT) of the transformed shape may be used to restore the area; but, $V$ or $v$ will remain changed. Therefore, a CT applied to the Clifford torus leads in general to a nonaxisymmetric shape with different relative volume $v \neq v^{*}$ but the same energy $F_{\kappa}=4 \pi^{2} \kappa$, which, for $v \neq v^{*}$, is lower than the energy of any of the axisymmetric shapes.

By discussing two limit cases, we now argue that the $v$ range accessible by CT's applied to the Clifford torus is given by $v^{*} \leq v<1$. First, consider special CT's with $\mathbf{R} \rightarrow\left(\mathbf{R} / R^{2}+\mathbf{a}\right) /\left(\mathbf{R} / R^{2}+\mathbf{a}\right)^{2}$ which consist of the composition of an inversion with a translation about the vector $\mathbf{a}$ and a second inversion. For small $\mathbf{a}$, the change in reduced volume can be expanded, as derived generally in Ref. 22. This leads for the Clifford torus to $v(\mathbf{a})-v^{*}$ $\approx c \mathbf{a}_{\text {perp }}^{2}$, where $c>0$ and $\mathbf{a}_{\text {perp }}$ is the component of $\mathbf{a}$ perpendicular to the axis of symmetry. This result shows that the special CT increases the relative volume $v$ for small a. Second, consider an inversion of the Clifford torus in a sphere whose center approaches the surface of the torus. In this limit, the transformed shape becomes a sphere with a small handle and has a relative volume $v$ that goes to 1 . Combining both results, one may conclude that for any $v$ in the range of $v^{*}<v<1$ a toroidal shape exists which is obtained by a CT of the Clifford torus. Therefore (if Willmore's conjecture is true), the shape of lowest bending energy is nonaxisymmetric for $v^{*} \leq v<1$.

In this region of the phase diagram, the three indepen- 
dent special CT's which correspond to the three linear independent choices of the translation vector a can be regarded as zero-energy deformation modes. Since the constraint on $v$ provides one relation for the amplitudes of these modes, there remain two "Goldstone modes" which conserve $v$ and cost no energy: The conformal symmetry leads to a twofold continuous degeneracy of the ground state. ${ }^{23}$

The phase diagram for shapes of toroidal topology and the type of transitions between them can be read off from Fig. 2. ${ }^{24}$ Start at small $v$ with a sickle-shaped toroid. With increasing $v$, this shape reaches, at $v=v_{M}^{\text {sic }}$, the limit of metastability and undergoes a discontinuous transition to the axisymmetric circular toroid, which subsequently, at $v=v^{*}$, becomes nonaxisymmetric via a continuous transition.

It is interesting to compare the energy $F_{K}$ of toroidal and spherical shapes ${ }^{15}$ for the same $v$. For $0<v \lesssim 0.72$, the toroidal ground state has lower energy than the spherical one, while for $0.72 \lesssim v \leq 1$, the ground state of spherical topology, as given by the prolate branch, has lower energy than $4 \pi^{2} \kappa$, which is the toroidal minimum in this range. Of course, the full vesicle energy $F$ also includes the Gaussian curvature energy $F_{\kappa_{G}}$, which contributes $4 \pi \kappa_{G}$ for shapes of spherical topology. The comparison between classes of different topology now depends on the nonuniversal value of $\kappa_{G}$, which favors toroidal shapes for $\kappa_{G}>0$. However, even for widely used membranes, neither the sign nor the value of $\kappa_{G}$ seems to be known.

While the bending energy (1) constitutes a minimal model for vesicle shapes, a more refined description may require either the inclusion of a spontaneous curvature $C_{0}$ [i.e., $(2 H)^{2}$ in (1) is replaced by $\left.\left(2 H-C_{0}\right)^{2}\right]^{9}$ or an additional constraint for the integrated mean curvature which defines the so-called bilayer coupling model. ${ }^{12,17}$ As will be shown elsewhere, these extensions of the model do not alter the main results of this paper, i.e., the existence of three branches of axisymmetric toroidal shapes and the dominance of nonaxisymmetric shapes over a large range of parameters. However, for nonzero spontaneous curvature, the two Goldstone modes gain a "mass," i.e., an energy which behaves like $\sim \kappa C_{0}^{2} A$ for small $C_{0}$. If it were possible to scan through a range of spontaneous curvatures, e.g., by adding different chemical ingredients to the solution, while keeping the osmolarity and, thus, $v$ fixed, the point at which $C_{0}$ changes sign should be reflected in enhanced fluctuations (which may well be overdamped due to hydrodynamic effects).

So far, the minimum of $F_{\kappa}$ for given $v$ has been considered. On long time scales, water can permeate through the membrane and the vesicle assumes the shape which minimizes $F_{\kappa}$ with respect to $v$. This minimum is characterized by $P=0$, since from Eq. (2), $P=-\left(\partial F_{\kappa} /\right.$ $\partial V)_{A, C_{0}}$ where the derivative is taken along a branch of stationary shapes. For $C_{0}=0$, the Clifford torus and its conformal transformations correspond to such a minimum. For $C_{0} \neq 0$, the Clifford torus solves (2) with $P<0$ for $C_{0}>0$ and $P>0$ for $C_{0}<0$, respectively. ${ }^{25,26}$ This shows that the shape of lowest $F_{\kappa}$ for $C_{0} \neq 0$ is not given by the Clifford torus but rather by a shape with a relative volume $v<v^{*}$ for $C_{0}>0$ and $v>v^{*}$ for $C_{0}<0$, respectively.

We close with a comment on the toroidal shapes recently observed for partially polymerized membranes. ${ }^{18}$ In this experiment, polymerizable but yet unpolymerized membranes were first cooled below the chain melting temperature $T_{m}$, which leads to tubular structures. This sample was then partially polymerized by UV irradiation and finally reheated above $T_{m}$. After this preparation, several toroidal shapes were observed, which were stable over days. Surprisingly, nearly all shapes seem to have had a ratio of radii characteristic of the Clifford torus. Although this nice observation demonstrates the existence of toroidal shapes, its quantitative analysis in terms of the model for fluid membranes discussed here is questionable. First, one has to suppose that the essential effect of the polymerization results in an effective spontaneous curvature $C_{0}$. Second, if $C_{0}$ is large and negative as suggested in Ref. 18, the shape of lowest bending energy is no longer the Clifford torus but rather a nonaxisymmetric shape with $v>v^{*}$. If the corresponding spontaneous curvature is small and distributed about zero, one would expect that apart from the Clifford torus also nonaxisymmetric shapes occur. This seems to have happened only in rare cases. The available experimental data, thus, seem to favor the conclusion that additional effects due to the polymerization, which are not yet included in the model discussed here, somehow single out the Clifford torus. Clearly, more experimental and theoretical work for this type of membrane is required to settle this important issue.

In summary, the theoretical study of toroidal shapes for fluid vesicles has revealed (i) three different branches of axisymmetric shapes, (ii) a large region of nonaxisymmetric shapes obtained by conformal transformations, and (iii) shape fluctuations of low energy for small spontaneous curvature.

Stimulating discussions with R. Lipowsky, E. Sackmann, and M. Wortis are gratefully acknowledged. I thank $\mathrm{M}$. Wortis for a careful reading of the manuscript and M. Mutz and D. Bensimon for sending me their preprint prior to publication. This work was funded in part by the Natural Sciences and Engineering Research Council of Canada.

Note added.-After submission of this paper, the observation of fluid vesicles with toroidal topology which indeed exhibit nonaxisymmetric shapes has been reported. ${ }^{27}$

${ }^{1}$ See, e.g., Statistical Mechanics of Membranes and Surfaces, edited by D. Nelson, T. Piran, and S. Weinberg, (World 
Scientific, Singapore, 1989); Proceedings of the International Conference on Geometry and Interfaces, Aussois, France, 1990 [J. Phys. (Paris), Colloq. 57, C7 (1990)].

${ }^{2}$ E. Sackmann, H. P. Duwe, and H. Engelhardt, Faraday Discuss. Chem. Soc. 81, 281 (1986).

${ }^{3}$ E. Evans and W. Rawicz, Phys. Rev. Lett. 64, 2094 (1990).

${ }^{4}$ M. B. Schneider, J. T. Jenkins, and W. W. Webb, J. Phys. (Paris) 45, 1457 (1984).

${ }^{5}$ H. Engelhardt, H. P. Duwe, and E. Sackmann, J. Phys. (Paris), Lett. 46, L395 (1985).

${ }^{6}$ J. F. Faucon, M. D. Mitov, P. Méléard, I. Bivas, and P. Bothorel, J. Phys. (Paris) 50, 2389 (1989).

${ }^{7}$ K. Berndl, J. Käs, R. Lipowsky, E. Sackmann, and U. Seifert, Europhys. Lett. 13, 659 (1990).

${ }^{8}$ P. B. Canham, J. Theoret. Biol. 26, 61 (1970).

${ }^{9}$ W. Helfrich, Z. Naturforsch. 28c, 693 (1973).

${ }^{10}$ H. J. Deuling and W. Helfrich, J. Phys. (Paris) 37, 1335 (1976).

${ }^{11}$ M. A. Peterson, J. Appl. Phys. 57, 1739 (1985).

${ }^{12}$ S. Svetina and B. Zeks, Eur. Biophys. J. 17, 101 (1989).

${ }^{13}$ Ou-Yang Zhong-can and W. Helfrich, Phys. Rev. Lett. 59, 2486 (1987).

${ }^{14}$ S. T. Milner and S. A. Safran, Phys. Rev. A 36, 4371 (1987).

${ }^{15}$ U. Seifert, K. Berndl, and R. Lipowsky, Phys. Rev. A (to be published).

${ }^{16}$ L. Miao, B. Fourcade, M. Rao, M. Wortis, and R. K. P. Zia, Phys. Rev. A (to be published).

${ }^{17}$ E. Evans, Biophys. J. 14, 923 (1974).

${ }^{18} \mathrm{M}$. Mutz and D. Bensimon (to be published).

${ }^{19}$ In a related situation, the topology-changing shape transformation from a necklace of vesicles to single vesicles has suc- cessfully been induced by photodissociation of certain amphiphiles embedded in the membrane. See H. P. Duwe, P. Eggl, and E. Sackmann, Angew. Makromol. Chem. 166\& 167, 1 (1989). Likewise, (pulsed) electric fields can induce pore formation. See, e.g., D. C. Chang, Biophys. J. 56, 641 (1989).

${ }^{20}$ T. J. Willmore, Total Curvature in Riemannian Geometry (Ellis Horwood Ltd., Chicester, 1982). Evidence for the Willmore conjecture can be found in Sect. 5.3 and Chap. 6 of this monograph.

${ }^{21}$ B. Duplantier, Physica (Amsterdam) 168A, 179 (1990); B. Duplantier, R. E. Goldstein, V. Romero-Rochin, and A. I. Pesci, Phys. Rev. Lett. 65, 508 (1990).

${ }^{22}$ U. Seifert (to be published).

${ }^{23}$ The possibility of continuous conformal fluctuations has previously been pointed out in Ref. 21 .

${ }^{24}$ Whether nonaxisymmetric shapes which are not obtained by CT's become relevant for $v<v^{*}$ can only be investigated by a numerical stability analysis of the axisymmetric shapes, which has not been attempted so far.

${ }^{25} \mathrm{Ou}$-Yang Zhong-can, Phys. Rev. A 41, 4517 (1990).

${ }^{26}$ Concerning the local stability of the Clifford torus, a close inspection of Ref. 25 reveals that the stability criterion $C_{0}$ $\lesssim-3.9(4 \pi / A)^{1 / 2}$ derived there yields only a sufficient but $a$ not necessary condition. On the other hand, one can prove, using CT's within the framework developed in Ref. 22, that the Clifford torus is locally unstable for $C_{0}>0$. Thus, the upper bound $C_{0}^{*}$ for stability obeys $-3.9(4 \pi / A)^{1 / 2} \leqslant C_{0}^{*} \leq 0$. The precise determination of $C_{0}^{*}$ requires a numerical stability analysis not attempted so far.

${ }^{27} \mathrm{D}$. Bensimon, in Proceedings of the Workshop on Dynamical Phenomena at Interfaces, Surfaces, and Membranes, Les Houches, 18-28 February 1991 (to be published). 